\title{
SINGLE-POINT BLOW-UP FOR A SEMILINEAR REACTION-DIFFUSION SYSTEM
}

\author{
NeJiB MAHMOUdi
}

Abstract. In this paper, we consider positive solutions of the system

$$
u_{t}-\Delta u=u^{r} v^{p}, \quad v_{t}-\Delta v=u^{q} v^{s}
$$

$t \in(0, T), x \in \mathbf{B}(0, R)=\left\{x \in \mathbb{R}^{n}|| x \mid<R\right\}$ or $x \in \mathbb{R}^{n}$ and $p, q, r, s>1$. We prove single-point blow-up if $r<q+1$ and $s<p+1$ and for a large class of radial decreasing solutions. This extends the result of Friedman and Giga for this basic system known only for $p=q=r=s$. We also obtain lower pointwise estimates for the blow-up profiles.

Mathematics subject classification (2010): 35B20, 35B40, 35B50, 35K55, 35K57, 35K58.

Keywords and phrases: nonlinear parabolic equations, reaction-diffusion equations, semilinear parabolic equations, asymptotic behavior of solutions, single-point blow-up.

\section{REFERENCES}

[1] H. Chen, Global existence and blow-up for a nonlinear reaction-diffusion system, J. Math. Anal. Appl., 212 (1997), 481-492.

[2] H. Chen, Blow-up estimates of positive solutions of a reaction-diffution system, Fourth Int. Conf. Dyn. Sys. Dif. Eq. May 24-27, 2002, Wilmington, NC, USA, 182-188.

[3] X.Y. Chen, H. Matano, Convergence, asymptotic periodicity, and finite-point blow-up in onedimensional semilinear heat equations, J. Differ. Equations, 78 (1989), 160-190.

[4] M. Escobedo, H.A. LeVINE, Critical blow-up and global existence numbers for a weakly coupled system of reaction-diffusion equations, Arch. Rational Mech. Anal., 129 (1995), 47-100.

[5] A. Friedman, Y. GigA, A single point blow-up for solutions of semilinear parabolic systems, J. Fac. Sci. Univ. Tokyo Sec. IA. Math., 34 (1987), 65-79.

[6] A. FRIEDmAn, B. MCLEOD, Blow-up of positive solution of semilinear heat equations, Indiana University Mathematics Journal, 34 (1985), 425-447.

[7] J.S. Guo, S. SaSAYAma, C.J. WANG, Blow-up rate estimate for a system of semilinear parabolic equations, Commun. Pure Appl. Anal., 8 (2009), 711-718.

[8] O.A. LADYŽEnSKAJA, V.A. SOlonNikov, N.N. URAL'CEVA, Linear and quasilinear equations of parabolic type, Amer. Math. Soc., Transl. Math. Monographs, Providence, R.I., 1968.

[9] P. Quittner, Ph. Souplet, Superlinear Prabolic Problems Blow-up, Global Existence and Steady States, Birkhauser Verlag AG, Bassel Boston Berlin, 2007.

[10] PH. Souplet, Single-point blow-up for a semilinear parabolic system, J. Eur. Math. Soc., 11 (2009), $169-188$.

[11] M. WANG, Blow-up estimates for a semilinear reaction diffusion system, J. Math. Anal. Appl., 257 (2001), 46-51.

[12] S. ZHENG, Global existence and global non-existence of solutions of reaction-diffusion system, Nonlinear Anal., 39 (2000), 327-340. 